\title{
BMJ Open Disease composition and epidemiological characteristics of primary care visits in Pudong New Area, Shanghai: a longitudinal study, 2016-2018
}

Xin Gong, ${ }^{1}$ Xiaoli Wang, ${ }^{2}$ Tianxing Shi, ${ }^{2}$ Jianwei Shi $(1),{ }^{3}$ Wenya Yu, ${ }^{3}$ Liang Zhou, ${ }^{3}$ Ning Chen, ${ }^{4}$ Jiaoling Huang (D) , ${ }^{3,5}$ Zhaoxin Wang (D) ${ }^{3}$

To cite: Gong X, Wang X, Shi T, et al. Disease composition and epidemiological characteristics of primary care visits in Pudong New Area, Shanghai: a longitudinal study, 2016-2018. BMJ Open 2020;10:e040878. doi:10.1136/ bmjopen-2020-040878

- Prepublication history and supplemental material for this paper is available online. To view these files, please visit the journal online (http://dx.doi org/10.1136/bmjopen-2020040878).

$\mathrm{JH}$ and ZW contributed equally.

Received 25 May 2020

Revised 20 0ctober 2020

Accepted 26 0ctober 2020
Check for updates

(C) Author(s) (or their employer(s)) 2020. Re-use permitted under CC BY-NC. No commercial re-use. See rights and permissions. Published by BMJ.

For numbered affiliations see end of article.

\section{Correspondence to}

Dr Jiaoling Huang;

jiaoling_huang@sina.com and

Dr Zhaoxin Wang;

supercell002@sina.com

\section{ABSTRACT}

Objectives This study aims to analyse the disease composition of primary care visits rather than specialist visits, the former of which had scarcely been studied. We adopted specific disease classification (International Statistical Classification of Diseases and Related Health Problems, 10th Revision), disease system and communicable/non-communicable/injury disease classification, and variations of sex and age were also analysed.

Setting We extracted data from all community health service centres (CHSCs) and community health service stations in Pudong, Shanghai, from 2016 to 2018 using the electronic health record systems of the Pudong health information centre.

Participants Our data included all 46720972 primary care visits from 2016 to 2018 in CHSCs in Pudong. Results We found that the top five diseases in primary care visits continued to be primary hypertension, problems related to medical facilities, chronic ischaemic heart disease, unspecified diabetes mellitus and acute upper respiratory infection. Lipoprotein metabolism disorder visits continued to increase over the study years. The numbers and proportions of patients with hypertension and unspecified diabetes were higher among men than women, and other cerebrovascular diseases were higher among women than men. The top five disease systems were circulatory system diseases, respiratory system diseases, endocrine/nutritional/metabolic diseases, factors influencing health status and digestive system diseases. The rankings of respiratory system and endocrine/nutritional/metabolic diseases rose over time. Non-communicable diseases (NCDs) accounted for approximately $90 \%$ of the primary care visits-a much higher percentage than other causes. The top five NCDs in primary care visits were cardiovascular and circulatory diseases, musculoskeletal disorders, diabetes, digestive diseases and urogenital diseases. Compared with women, men suffered from cardiovascular diseases at an earlier age.

Conclusions Different from specialist visits, common diseases, especially NCDs, were the main disease composition of current primary healthcare visits while the former focused on intractable diseases such as tumours, indicating that primary healthcare had played the role of gatekeeper of the healthcare system.
Strengths and limitations of this study

- The study examined the realisation status of hierarchical diagnosis and treatment based on primary care in Shanghai using the electronic medical data of all community health service centres in Pudong region.

- This is the first community-level analysis of the epidemiological disease characteristics of primary care visits in the entire Pudong region.

- Limited by the electronic medical record system, we analysed the disease composition only at the primary care level, rather than including both specialist visits and primary care visits at the same time, and future work should also consider examining the latest data after 2018.

\section{BACKGROUND}

Scholars worldwide agree that general practitioners (GPs) have a significant effect on improving population health and controlling medical expenses. ${ }^{1-5}$ Additionally, it is widely accepted that an orderly medical treatment system should be based on GPs. Many scholars argued that it would not be possible to achieve first-contact GPs and 'GP-specialist' referrals if GPs could not provide satisfactory primary healthcare, and therefore orderly medical care could not be achieved as well. ${ }^{67}$ The World Bank estimated that up to $90 \%$ of healthcare demands could potentially be dealt with at the primary care level. ${ }^{8}$ In China, primary healthcare was set as one of five targets in the new round of healthcare reform in 2009. The Chinese government initially intended to improve access to medical care and decrease individual medical costs by implementing a GP system comprising the main body of tier 1 hospitals. ${ }^{9}$ In China, healthcare is provided through a three-tiered system in which all specialists work in secondary and tertiary hospitals, 
whereas GPs make up the majority of the staff of tier 1 hospitals-community health service centres (CHSCs) or community health service stations (CHSSs) in cities and medical clinics in rural areas. ${ }^{10}$ The family doctor (FD) system was initially established throughout China in 2013. ${ }^{11}$ A referral system was proposed by the central government in 2015, with the key strategy of strengthening the primary healthcare system. ${ }^{12}$ A series of policies have been implemented to encourage people to visit GPs in CHSC for their initial visit; these policies include free health examinations, lower out-of-pocket medical expenses and more personalised health management services. $^{13}$

However, some researchers have argued that patients continue their previous doctor-visiting behaviour, preferring visiting specialists directly, regardless of the severity of their conditions. ${ }^{14} \mathrm{Li}$ et at explored the primary healthcare system in China thoroughly, pointing out that patients still preferred to go to hospitals for more specialised consultations rather than first-contact FDs in CHSCs; these scholars argued that the reason for this behaviour could be found on the supply side (ie, FDs lacked adequate technical support and appropriate financial incentives to deliver integrated and high-quality care). ${ }^{9}$ Other studies have found that the FD system has played a positive role in achieving orderly doctor-visiting behaviour, reporting that most common diseases were being treated through the primary healthcare system. ${ }^{15-17} \mathrm{Li}^{18}$ argued that, although the experience in Shanghai City, Zhejiang Province, Qinghai Province and Zhengjiang City in Jiangsu Province had been endorsed by the central government, the policy innovations in Xiamen City had also succeeded in alleviating the overutilization of secondary care with improved outcomes in medical treatment and chronic disease management. ${ }^{18} \mathrm{Li}$ et $a l^{19}$ demonstrated that local health insurance coverage could help improve the management and control of hypertension in a primary care setting. A longitudinal study conducted by Huang et $a l^{20}$ suggested that the percentage of patients preferring to first contact FDs in CHSCs or CHSSs had progressively increased. However, there is still significant controversy regarding the role of FDs in achieving the orderly coordination of medical care through the referral system.

The present study explored a wide disease spectrum. It is generally accepted that the main diseases affecting human health have transitioned from acute and chronic infectious diseases to chronic non-communicable diseases (NCDs). ${ }^{21}$ NCDs is already the main trend of epidemiology, although there will be outbreaks of epidemic diseases from time to time, such as the recent global transmission of COVID-19. ${ }^{22}$ The WHO has identified NCDs as the leading cause of death globally, ${ }^{23}$ and numerous studies in China have also revealed the same dramatic epidemiological shift. ${ }^{24}$ However, previous studies in China have several limitations. Many of these studies have selected one or a few hospitals to explore epidemiological characteristics and the disease spectrum due to data collection problems, ${ }^{25}$ and existing studies have tended to focus on inpatient diseases, neglecting epidemiological characteristics at the primary care level. ${ }^{26-29}$ Huang et $a l^{30}$ analysed the existing studies of the disease spectrum and epidemiological characteristics, finding that $39.1 \%$ of these studies focused on inpatients, $20.4 \%$ on emergency room patients, $9.1 \%$ on literature and statistical reports, and $7.2 \%$ on medical examinations, with epidemiological surveys accounting for only $6.4 \%$ of current studies. No existing studies on this topic have focused on primary care visits or included data on entire regions. We believe that it is significant and necessary to fill this research gap, overcoming the potential bias of investigating a single hospital or tertiary hospitals, exploring the epidemiological characteristics of primary care visits, and contributing to understanding the controversial topic of the role of FDs in the orderly coordination of medical care.

In this study, we tried to map the disease composition of primary care visits by collecting real data for the whole district of Pudong New Area of Shanghai using the 'big data' of 46720972 visit records, to analyse the epidemiological characteristics at the primary care level, to explore the variation of disease distribution by sex and age over years, and to summarise epidemiological characteristics by comparing with that of specialist visits in China and other countries.

\section{METHODS}

\section{Study design and setting}

This study was conducted in the Pudong New Area district of Shanghai. As the largest district of Shanghai, Pudong has a permanent population of 5.5 million inhabitants and covers an area of 1210 square kilometres. ${ }^{31}$ Pudong is always the pilot area for healthcare reform in Shanghai and even in China, and many policies have been piloted and implemented initially in Pudong. In Shanghai, only CHSCs and CHSSs provide primary healthcare services, rather than specialists in tier-2 and tier-3 hospitals, and the CHSSs are affiliated to CHSCs. Pudong has the largest number of CHSCs than any other district in Shanghai. The 47 CHSCs and 386 CHSSs in Pudong provide more than 15 million visits per year. All 47 CHSCs and CHSSs belong to the Health Commission of Pudong New Area, and the medical data are managed by the health information centre (HIC) affiliated to the Health Commission. Since 2014, we have cooperated with the HIC to extract the whole data of primary visits in Pudong New Area. Thus, this is a population-based study covering all primary care visits using longitudinal data from 2016 to 2018.

\section{Data collection}

Data on primary care visits from 2016 to 2018 were extracted from Pudong HIC's electronic health records (EHRs) system. We chose 2016 as the starting point because the system was not completely reliable until 2016, even though the EHR system including data of all primary care visits had been launched by the Pudong HIC since 2014. We used data of all 46720972 
primary care visits recorded from 2016 to 2018 in all 47 CHSCs in Pudong (17 008874 visits in 2016, 14670 104 visits in 2017 and 15041994 visits in 2018). The data included all outpatient visits from all the 47 CHSCs and the 386 CHSSs in Pudong, regardless of whether they had medical insurance or a type of medical insurance, whether they were permanent residents or not. However, the data didn't include the unofficial care clinics. In Shanghai, there were few unofficial care clinics for GPs and patients rarely visited the unofficial clinics, according to the Shanghai Pudong New Area statistical yearbook. ${ }^{32}$

The data from the EHR system consisted of diagnosis information, institution name, visit time, International Statistical Classification of Diseases and Related Health Problems, 10th Revision (ICD-10) diagnostic codes, sex and age. The physicians diagnosed each patient according to ICD-10 codes. The diagnosis would be problems related to medical facilities (Z75) if the patients only visited for health examination. Original electronic documents were reviewed and audited by the HIC, and the original data were extracted and analysed. We received the data without personal information so that no individual patient could be identified.

\section{Measures}

We initially analysed the data using ICD-10 codes, and then classified the diseases by the ICD-10 system according to its 2016 version. We focused on NCDs because NCDs accounted for a large proportion of diseases and also because of the enormous global burden associated with NCDs. The ICD-10 system codes for disease systems and NCDs can be found in online supplemental tables 1-3. The data were analysed separately by sex (male and female) and age groups. The population was divided into five age groups: $\leq 18$ years, $19-39$ years, $40-59$ years, $60-79$ years and $\geq 80$ years.

\section{Statistical analysis}

We analysed all data using SAS software V.9.20. The most common disorders and systems and all NCDs were ranked by proportion of primary care visits. First, the disease composition of the overall population was analysed. The sex and age subgroups were then analysed separately. Finally, we conducted a cross analysis of both sex and age to further investigate the disease composition of primary care. No statistical tests were used in our study because this is a descriptive study using longitudinal data from the whole population rather than a sample data.

\section{Patient and public involvement}

We use secondhand statistical data, rather than an original individual data set, which was reviewed and processed anonymously by the Health Commission. Thus, patients and the public were not required to be directly involved in this study.

\section{RESULTS}

Ranking of disorders in all primary care visits within sex and age groups

The order of the top five diseases did not change over time among the patients who visited CHSCs from 2016 to 2018: The most common diseases were hypertension, problems related to medical facilities, chronic ischaemic heart disease, unspecified diabetes mellitus, and acute upper respiratory infections of multiple and unspecified sites. The proportions of patients with lipoprotein metabolism disorder, conjunctivitis and other arrhythmias continually increased over the three study years (figure 1). In each year, the numbers and proportions of patients with hypertension and unspecified diabetes were higher among men than women. Conversely, the numbers and proportions of patients with chronic ischaemic heart disease, sequelae of cerebrovascular disease and other cerebrovascular diseases were higher among women than men (see figure 2 for details).

Figure 3 presents the disease composition by age groups. In each adult age group, the proportions increased and the rankings rose with age for ischaemic heart disease, cerebrovascular sequelae and other diseases, whereas the proportion decreased and the rankings dropped with age for acute upper respiratory infection. Cross analysis of both sex and age revealed that, in the 19-39 years age group, the proportion for hypertension was much lower among women than men. Among the three oldest age groups, the proportion for ischaemic heart disease increased with age more obviously for women than for men (see online supplemental table 1 for details).

\section{Distribution of disease systems overall and by age and sex groups}

As shown in figure 4, circulatory system diseases, factors influencing health status and contact with health services, respiratory system diseases, endocrine diseases/nutrition/ metabolism diseases, and digestive system diseases were the top five disease types, accounting for most primary care visits in each study year $(77.1 \%$ in $2016,75.51 \%$ in 2017 and $76.76 \%$ in 2018). The proportions of circulatory system diseases, endocrine diseases, nutrition and metabolism diseases, and urogenital system diseases increased each year. There were some differences between women and men. In each year, the proportions of visits due to circulatory system diseases in men increased and were much higher than those of women (see figure 5 for details).

The analysis by age group (figure 6) showed that the proportion of respiratory system diseases was far higher among patients aged 19-39 years than in the other age groups. In the 60-79 years age group, the proportions of endocrine diseases and nutritional and metabolic diseases were much higher than in other age groups. In addition, among the three oldest age groups, the proportion of diseases of the circulatory system, the most common disease system, increased with age. The difference between men and women was largest in the 19-39 years age group, for example, the top three disease systems for men were 


\begin{tabular}{|c|c|c|c|c|c|c|c|c|}
\hline Visits & Proportion & Rank in 2016 & Visits & Proportion & Rank in 2017 & Visits & Proportion & Rank in 2018 \\
\hline 3398395 & $5 \quad 19.98 \%$ & 1 primary hypertension & 2847164 & $19.41 \%$ & 1 primary hypertension & 3154259 & $20.97 \%$ & 1 primary hypertension \\
\hline 2600152 & $15.29 \%$ & 2 problems related to medical facilities & 1522385 & $10.38 \%$ & 2 problems related to medical facilities & 1381193 & $9.18 \%$ & 2 problems related to medical facilities \\
\hline 1432446 & $8.42 \%$ & 3 chronic ischaemic heart disease & 1382182 & $9.42 \%$ & 3 chronic ischaemic heart disease & 1315727 & $8.75 \%$ & 3 Chronic ischaemic heart disease \\
\hline 1168805 & $6.87 \%$ & 4 unspecified diabetes mellitus & 959294 & $6.54 \%$ & 4 unspecified diabetes mellitus & 1018359 & $6.77 \%$ & 4 unspecified diabetes mellitus \\
\hline 680753 & $4.00 \%$ & 5 acute upper respiratory infection & 582887 & $3.97 \%$ & 5 acute upper respiratory infection & 696802 & $4.63 \%$ & 5 acute upper respiratory infection \\
\hline 474266 & $2.79 \%$ & 6 gastritis and duodenitis & 426415 & $2.91 \%$ & 6 gastritis and duodenitis & 464613 & $3.09 \%$ & 6 disorders of lipoprotein metabolism \\
\hline 332220 & $1.95 \%$ & 7 other arthritis & 391373 & $2.67 \%$ & 7 disorders of lipoprotein metabolism & 394928 & $2.63 \%$ & 7 gastritis and duodenitis \\
\hline 325031 & $1.91 \%$ & 8 bronchitis, not specified & 349013 & $2.38 \%$ & 8 sequelae of cerebrovascular disease & 323484 & $2.15 \%$ & 8 sequelae of cerebrovascular disease \\
\hline 323769 & $1.9 \%$ & 9 disorders of lipoprotein metabolism & 290211 & $1.98 \%$ & 9 other arthritis & 269845 & $1.79 \%$ & 9 other arthritis \\
\hline 319338 & $1.88 \%$ & 10 sequelae of cerebrovascular disease & 283766 & $1.93 \%$ & 10 bronchitis, not specified & 268780 & $1.79 \%$ & 10 bronchitis, not specified \\
\hline 248693 & $1.46 \%$ & 11 Dorsalgia & 210260 & $1.43 \%$ & 11 Other functional intestinal disorders & 220305 & $1.46 \%$ & 11 Other functional intestinal disorders \\
\hline 192310 & $1.13 \%$ & 12 Other functional intestinal disorders & 192338 & $1.31 \%$ & 12 Hyperplasia of prostate & 219683 & $1.46 \%$ & 12 sleep disorders \\
\hline 186552 & $1.1 \%$ & 13 Other dermatitis & 182201 & $1.24 \%$ & 13 Other dermatitis & 217047 & $1.44 \%$ & 13 Hyperplasia of prostate \\
\hline 184809 & $1.09 \%$ & 14 Acute bronchitis & 174191 & $1.19 \%$ & 14 Other cerebrovascular diseases & 177900 & $1.18 \%$ & 14 Conjunctivitis \\
\hline 173350 & $1.02 \%$ & 15 Hyperplasia of prostate & 163848 & $1.12 \%$ & 15 Conjunctivitis & 169585 & $1.13 \%$ & 15 Other dermatitis \\
\hline 173321 & $1.02 \%$ & 16 Gingivitis and periodontal diseases & 162345 & $1.11 \%$ & 16 Gingivitis and periodontal diseases & 166523 & $1.11 \%$ & 16 Other cerebrovascular diseases \\
\hline 165295 & $0.97 \%$ & 17 Other cerebrovascular diseases & 162318 & $1.11 \%$ & 17 Dorsalgia & 165455 & $1.1 \%$ & 17 Gingivitis and periodontal diseases \\
\hline 160144 & $0.94 \%$ & 18 Conjunctivitis & 150086 & $1.02 \%$ & 18 Acute bronchitis & 159118 & $1.06 \%$ & 18 Acute bronchitis \\
\hline 151382 & $0.89 \%$ & 19 Dizziness and giddiness & 143277 & $0.98 \%$ & 19 Other joint disorders & 151551 & $1.01 \%$ & 19 Other cardiac arrhythmias \\
\hline 150956 & $0.89 \%$ & 20 Other joint disorders & 138157 & $0.94 \%$ & 20 Osteoporosis without fracture & 141097 & $0.94 \%$ & 20 Osteoporosis without fracture \\
\hline \multirow[t]{3}{*}{125023} & $0.74 \%$ & 24 Osteoporosis without fracture & 124278 & $0.85 \%$ & 21 sleep disorders & 139733 & $0.93 \%$ & 21 Other joint disorders \\
\hline & & & 113329 & $0.77 \%$ & 24 Dizziness and giddiness & 126937 & $0.84 \%$ & 22 Dorsalgia \\
\hline & & & 51740 & $0.35 \%$ & 39 Other cardiac arrhythmias & & & \\
\hline
\end{tabular}

Figure 1 Distribution of the top 20 diseases in primary care visits, 2016-2018.

diseases of the respiratory system, factors influencing health status and contact with health services and diseases of the digestive system while they were factors influencing health status and contact with health services, diseases of the respiratory system and diseases of the genitourinary system for women (see online supplemental table 2 for details on the cross analysis of sex and age).

\section{NCDs overall and by age group and sex}

From 2016 to 2018, primary care visits for NCDs were much more frequent, compared with visits for other causes, with NCDs accounting for approximately $90 \%$ of all visits. This was followed by infectious diseases. Few differences were observed by sex and age groups over years. In 2016 and 2017, the top five NCDs were cardiovascular and

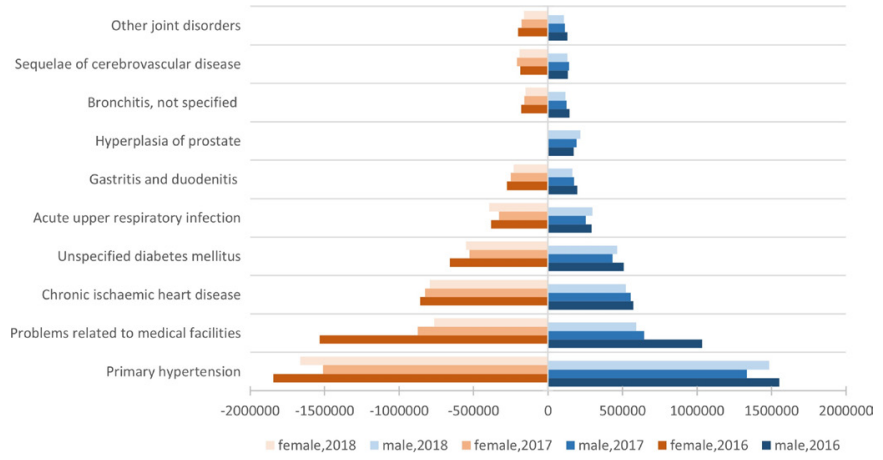

Figure 2 Distribution of the top 20 diseases in primary care visits by sex, 2016-2018. circulatory system diseases, musculoskeletal disorders, diabetes, digestive diseases and urogenital diseases. In 2018, diabetes replaced musculoskeletal disorders as the NCD with the second-highest number of primary care visits (see figure 7). In sex group analysis, the proportion of musculoskeletal disorders was more among women than among men while the proportion of diabetes was more among men (see table 1 for details).

The analysis by age group showed that the composition of NCDs was quite different for patients aged $\leq 18$ years, compared with the other age groups. The visits by patients aged 18 years or younger were commonly caused by respiratory diseases, oral diseases and skin diseases. Among the three oldest age groups (40-59 years, 60-79 years and $\geq 80$ years), cardiovascular and circulatory diseases accounted for the largest number of primary care visits, with a proportion far higher than those of the other diseases. Furthermore, in the three oldest age groups, the proportions of cardiovascular and circulatory diseases increased with age. The proportion of diabetes was also higher in the three oldest age groups than in the youngest age group ( $\leq 18$ years; see table 2 for details). The cross analysis of sex and age revealed a difference between men and women in patients aged 19-39 years. In this age group, visits by women were mainly caused by urogenital diseases, whereas cardiovascular and circulatory system diseases ranked first for visits by men. Among the three oldest age groups, the proportion of visits for cardiovascular and circulatory diseases ranked first for 


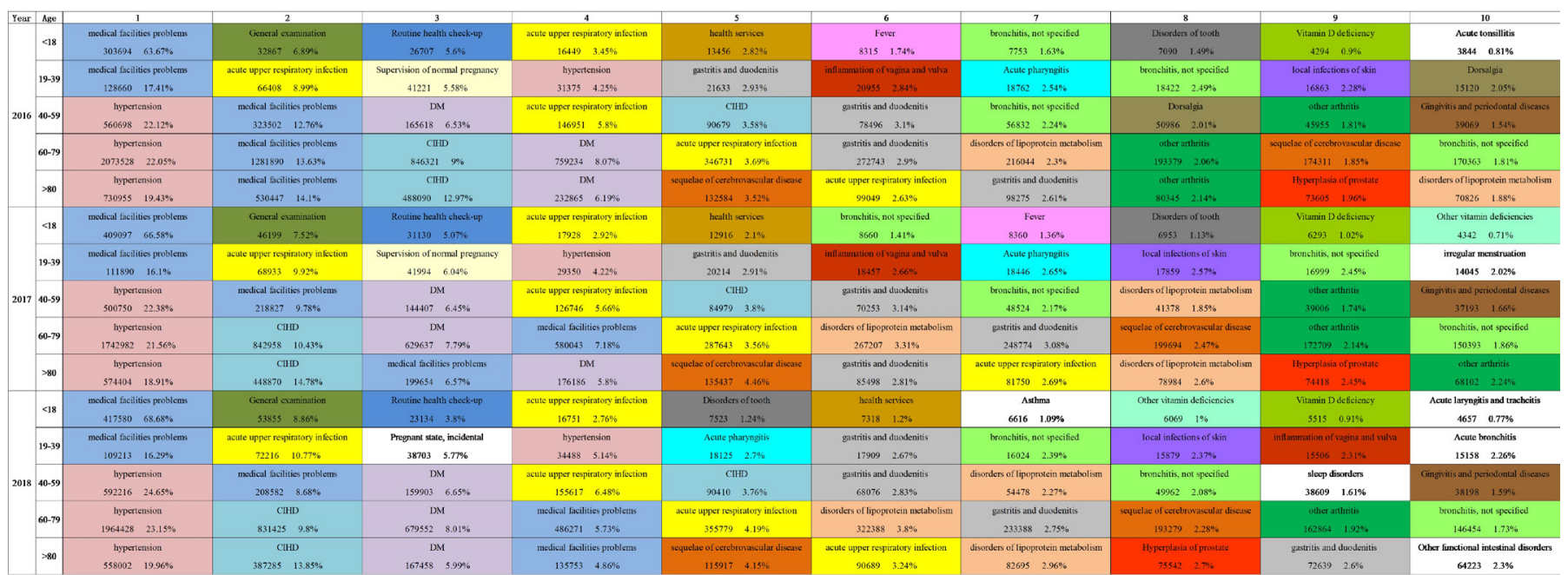

Figure 3 Distribution of the top 20 diseases in primary care visits by age group, 2016-2018. CHD, Coronary Heart Disease; DM, Diabetes Mellitus.

both men and women (see online supplemental table 3 for details).

\section{DISCUSSION}

We found that the top five diseases in primary care visits were consistently primary hypertension, problems related to medical facilities, chronic ischaemic heart disease, unspecified diabetes mellitus and acute upper respiratory infection. Lipoprotein metabolism disorder was found to increase continually over the study years, possibly because of an increased detection rate and a higher prevalence caused by changes in diet. ${ }^{33}$ The top five disease systems were circulatory system diseases, respiratory system diseases, endocrine/nutritional/metabolic diseases, factors influencing health status and digestive system diseases. Respiratory system and endocrine/nutritional/ metabolic diseases moved up in terms of their frequency ranking over the study years. More (and more serious) air pollution may have led to the increase in respiratory system diseases. ${ }^{34}$ Increases of both prevalence and detection rates likely led to the increases in endocrine/nutritional/metabolic diseases and lipoprotein metabolism

\begin{tabular}{|c|c|c|c|c|c|c|c|c|}
\hline Visits & Proportion & Rank in 2016 & Visits & Proportion & Rank in 2017 & Visits & Proportion & Rank in 2018 \\
\hline 5444113 & $32.01 \%$ & 1 Diseases of the circulatory system & 4888033 & $33.32 \%$ & 1 Diseases of the circulatory system & 5198248 & $34.56 \%$ & 1 Diseases of the circulatory system \\
\hline 2802353 & $16.48 \%$ & 2 Factors influencing health status & 1742896 & $6 \quad 11.88 \%$ & 2 Factors influencing health status & 1711014 & $11.37 \%$ & 2 Diseases of the respiratory system \\
\hline 1896546 & $6 \quad 11.15 \%$ & 3 Diseases of the respiratory system & 1608940 & $10.97 \%$ & 3 Diseases of the respiratory system & 1707278 & $11.35 \%$ & 3 Endocrine, nutritional and metabolic diseases \\
\hline 1624835 & $5 \quad 9.55 \%$ & 4 Endocrine, nutritional and metabolic diseases & 1530219 & $10.43 \%$ & 4 Endocrine, nutritional and metabolic diseases & 1638513 & $10.89 \%$ & 4 Factors influencing health status \\
\hline 1376850 & $8.09 \%$ & 5 Diseases of the digestive system & 1306600 & $8.91 \%$ & 5 Diseases of the digestive system & 1291378 & $8.59 \%$ & 5 Diseases of the digestive system \\
\hline 1256618 & $7.39 \%$ & 6 The musculoskeletal system diseases & 1133434 & $4 \quad 7.73 \%$ & 6 The musculoskeletal system diseases & 1107375 & $7.36 \%$ & 6 The musculoskeletal system diseases \\
\hline 677759 & $3.98 \%$ & 7 Diseases of the genitourinary system & 651190 & $4.44 \%$ & 7 Diseases of the genitourinary system & 651518 & $4.33 \%$ & 7 Diseases of the genitourinary system \\
\hline 532593 & $3.13 \%$ & 8 Symptoms and abnormal findings & 404614 & $2.76 \%$ & 8 Diseases of the skin & 393819 & $2.62 \%$ & 8 Diseases of the skin \\
\hline 421177 & $2.48 \%$ & 9 Diseases of the skin & 401698 & $2.74 \%$ & 9 Symptoms and abnormal findings & 327965 & $2.18 \%$ & 9 Diseases of the nervous system \\
\hline 278135 & $1.64 \%$ & 10 Diseases of the eye and adnexa & 291720 & $1.99 \%$ & 10 Diseases of the eye and adnexa & 312184 & $2.08 \%$ & 10 Diseases of the eye and adnexa \\
\hline 264096 & $1.55 \%$ & 11 Diseases of the nervous system & 229170 & $1.56 \%$ & 11 Diseases of the nervous system & 299233 & $1.99 \%$ & 11 Symptoms and abnormal findings \\
\hline 146039 & $0.86 \%$ & 12 Mental and behavioural disorders & 209240 & $1.43 \%$ & 12 Mental and behavioural disorders & 135325 & $0.9 \%$ & 12 Mental and behavioural disorders \\
\hline 113476 & $0.67 \%$ & 13 Infectious and parasitic diseases & 107823 & $0.73 \%$ & 13 Infectious and parasitic diseases & 102475 & $0.68 \%$ & 13 Infectious and parasitic diseases \\
\hline 65041 & $0.38 \%$ & 14 Diseases of ear and mastoid process & 70860 & $0.48 \%$ & 14 Diseases of ear and mastoid process & 75851 & $0.5 \%$ & 14 Diseases of ear and mastoid process \\
\hline 36040 & $0.21 \%$ & 15 Injury, poisoning & 36994 & $0.25 \%$ & 15 Diseases of blood and immune mechanism & 37635 & $0.25 \%$ & 15 Diseases of blood and immune mechanism \\
\hline 35164 & $0.21 \%$ & 16 Diseases of blood and immune mechanism & 26229 & $0.18 \%$ & 16 Injury, poisoning & 25587 & $0.17 \%$ & 16 Injury, poisoning \\
\hline 15451 & $0.09 \%$ & 17 External causes of morbidity and mortality & 12928 & $0.09 \%$ & 17 Neoplasms & 16549 & $0.11 \%$ & 17 Neoplasms \\
\hline 14214 & $0.08 \%$ & 18 Neoplasms & 11334 & $0.08 \%$ & 18 External causes of morbidity and mortality & 6220 & $0.04 \%$ & 18 Pregnancy, childbirth and the puerperium \\
\hline 7235 & $0.04 \%$ & 19 Pregnancy, childbirth and the puerperium & 5210 & $0.04 \%$ & 19 Pregnancy, childbirth and the puerperium & 3295 & $0.02 \%$ & 19 External causes of morbidity and mortality \\
\hline 1106 & $0.01 \%$ & 20 Congenital and chromosomal abnormalities & 934 & $0.01 \%$ & 20 Congenital and chromosomal abnormalities & 518 & $0.00 \%$ & 20 Congenital and chromosomal abnormalities \\
\hline 32 & $0.00 \%$ & 21 Certain conditions in the perinatal period & 38 & $0.00 \%$ & 21 Certain conditions in the perinatal period & 14 & $0.00 \%$ & 21 Certain conditions in the perinatal period \\
\hline 1 & $0.00 \%$ & 22 Codes for special purposes & 0 & $0.00 \%$ & 22 Codes for special purposes & 0 & $0 \%$ & 22 Codes for special purposes \\
\hline
\end{tabular}

Figure 4 Distribution of disease systems in primary care visits, 2016-2018. 


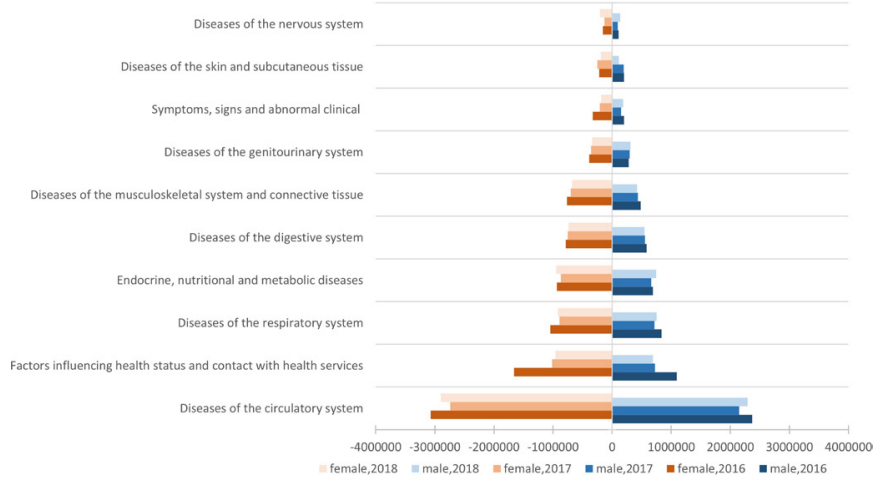

Figure 5 Distribution of disease systems in primary care visits by sex, 2016-2018.

disorders. Our findings for the disease composition of primary care visits are very different from those reported by recent studies of specialist visits in large hospitals. According to recent studies conducted in tier 2 or tier 3 hospitals, the most common top diseases among inpatients were neoplasms, circulatory diseases, respiratory diseases, factors affecting health status and nervous system diseases. ${ }^{35-39}$ In our study, we found some differences in the most common diseases for primary healthcare visits and specialist visits. The specific diseases varied more substantially, with CHSCs more often dealing with primary and common diseases. A preliminary conclusion could be drawn that primary care in Shanghai, at least in Pudong district, has played the role of gatekeeper, based on which an ordered treatment and referral system could be established.

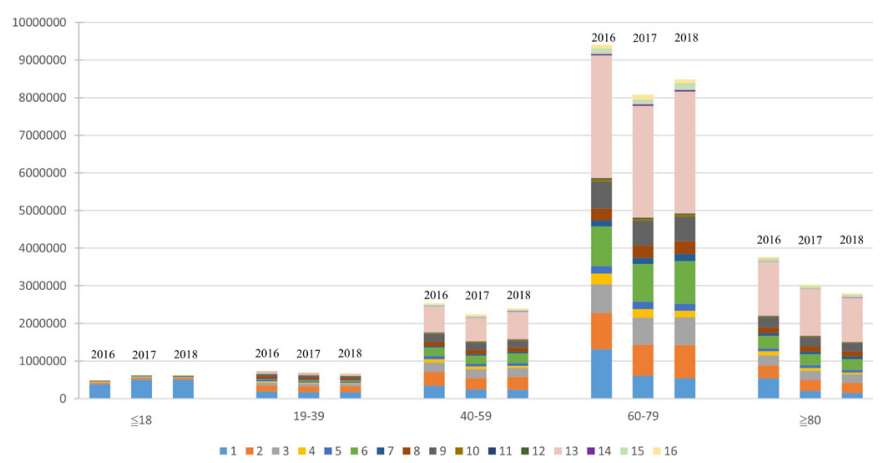

Figure 6 Distribution of disease systems in primary care visits by age group, 2016-2018. Note: (1) Factors influencing health status and contact with health services, (2) Diseases of the respiratory system, (3) Diseases of the digestive system, (4) Symptoms, signs and abnormal clinical and laboratory findings, (5) Diseases of the skin and subcutaneous tissue, (6) Endocrine, nutritional and metabolic diseases, (7) Diseases of the eye and adnexa, (8) Diseases of the genitourinary system, (9) Diseases of the musculoskeletal system and connective tissue, (10) Certain infectious and parasitic diseases, (11) Injury, poisoning and certain other consequences of external causes, (12) Diseases of the blood, blood-forming organs and the immune mechanism, (13) Diseases of the circulatory system, (14) Diseases of the ear and mastoid process, (15) Diseases of the nervous system, (16) Diseases of other systems.
We also found that visits for NCDs were dominant in terms of disease composition, accounting for approximately $90 \%$ of the primary care visits in our data set, suggesting an ongoing health transition. Recent studies have suggested that China has completed the epidemiological transition from a predominance of infectious diseases and maternal and perinatal conditions to chronic diseases and injury being the main conditions affecting human health. ${ }^{40}$ However, because China, like other developed countries, is facing challenging situations such as the impact of smoking, hypertension, the health effects of environmental pollution and the rise of HIV/AIDS, we prefer to describe the current situation in China as an ongoing 'health transition'. This designation considers more wide-ranging factors, including cultural, social and environmental determinants of health, rather than focusing only on improvements in medical care and public health. We found that the top five NCDs in our primary healthcare visits were cardiovascular and circulatory diseases, musculoskeletal disorders, diabetes, digestive diseases and urogenital diseases, which differed from recent reports on the disease composition in specialist visits. Shi $e t a l^{41}$ collected data from 12 public hospitals in China, finding that the top five NCDs were cardiovascular and circulatory diseases, urogenital diseases, chronic respiratory diseases, cancers and digestive diseases. ${ }^{41}$ The emergence of NCDs in China has received significant attention, and the prevalence and burden of NCDs continue to grow. Primary healthcare is thought to be an appropriate strategy to deal with such challenges, as has been strongly recommended by the WHO and implemented globally. ${ }^{42}$ Kane $e t ~ a t^{43}$ has argued that NCDs can be treated effectively through primary healthcare even in resource-poor settings. Besides, with the increase in the proportion of NCDs, the proportion of communicable diseases (CDs) and injuries had been declining over years. However, the disease composition of CDs, NCDs and injuries may be changed by the outbreak of COVID19 , but we believe that the COVID-19 outbreak is a mutation which will not change the long-term trend of disease composition.

We further analysed disease composition differences by sex and age and found that the numbers and proportions of patients with hypertension and unspecified diabetes were higher among men than women, whereas chronic ischaemic heart disease, sequelae of cerebrovascular disease and other cerebrovascular diseases were higher among women than men. Such sex differences in chronic ischaemic heart disease have been widely observed in current studies, but investigations and data are still lacking. Abbey and Stewart have pointed out that over the last 5 years ischaemic cardiac disease has become the highest cause of mortality for women in most Western countries, but gender difference studies were lacking. ${ }^{44}$ Similar ideas were expressed by Ciambrone and Kaski. They pointed out that despite the relatively high prevalence of coronary artery disease in women, there were little data on the investigation and treatment of ischaemic 


\begin{tabular}{|c|c|c|c|c|c|c|c|c|}
\hline Visits & Proportion & Rank in 2016 & Visits $P$ & Proportion & Rank in 2017 & Visits I & Proportion & Rank in 2018 \\
\hline 5444113 & $\begin{array}{l}3 \quad 44.63 \% \\
\end{array}$ & 1 Cardiovascular and circulatory & 4888033 & $43.23 \%$ & 1 Cardiovascular and circulatory & 5198248 & $84.08 \%$ & 1 Cardiovascular and circulatory \\
\hline 1256618 & $8 \quad 10.3 \%$ & 2 Musculoskeletal disorders & 1133434 & $10.02 \%$ & 2 Musculoskeletal disorders & 1128039 & $9.57 \%$ & 2 Diabetes \\
\hline 1232252 & $2 \quad 10.1 \%$ & 3 Diabetes & 1052139 & $9.31 \%$ & 3 Diabetes & 1107375 & $5 \quad 9.39 \%$ & 3 Musculoskeletal disorders \\
\hline 962695 & $7.89 \%$ & 4 Digestive diseases & 901994 & $7.98 \%$ & 4 Digestive diseases & 883621 & $7.49 \%$ & 4 Digestive diseases \\
\hline 652062 & $5.35 \%$ & 5 Urogenital diseases & 629993 & $5.57 \%$ & 5 Urogenital diseases & 631566 & $5.36 \%$ & 5 Urogenital diseases \\
\hline 641173 & $5.26 \%$ & 6 Chronic respiratory diseases & 593480 & $5.25 \%$ & 6 Chronic respiratory diseases & 597480 & $5.07 \%$ & 6 Chronic respiratory diseases \\
\hline 421177 & $3.45 \%$ & 7 Skin and subcutaneous diseases & 491881 & $4.35 \%$ & 7 Endocrine diseases & 583751 & $4.95 \%$ & 7 Endocrine diseases \\
\hline 414155 & $3.4 \%$ & 8 Oral disease & 404614 & $3.58 \%$ & 8 Skin and subcutaneous diseases & 407757 & $3.46 \%$ & 8 Oral disease \\
\hline 413011 & $3.39 \%$ & 9 Endocrine diseases & 404606 & $3.58 \%$ & 9 Oral disease & 393795 & $3.34 \%$ & 9 Skin and subcutaneous diseases \\
\hline 334464 & $2.74 \%$ & 10 Sensory organ diseases & 354687 & $3.14 \%$ & 10 Sensory organ diseases & 380102 & $3.22 \%$ & 10 Sensory organ diseases \\
\hline 264019 & $2.16 \%$ & 11 Neurological disorders & 229131 & $2.03 \%$ & 11 Neurological disorders & 327917 & $2.78 \%$ & 11 Neurological disorders \\
\hline 146021 & $1.2 \%$ & 12 Mental and behavioural disorders & 209206 & $1.85 \%$ & 12 Mental and behavioural disorders & 135245 & $1.15 \%$ & 12 Mental and behavioural disorders \\
\hline 10510 & $0.09 \%$ & 13 Other neoplasms & 8804 & $0.08 \%$ & 13 Other neoplasms & 9214 & $0.08 \%$ & 13 Other neoplasms \\
\hline 3704 & $0.03 \%$ & 14 Cancer & 4124 & $0.04 \%$ & 14 Cancer & 7335 & $0.06 \%$ & 14 Cancer \\
\hline 1106 & $0.01 \%$ & 15 Congenital anomalies & 934 & $0.01 \%$ & 15 Congenital anomalies & 518 & $0.00 \%$ & 15 Congenital anomalies \\
\hline
\end{tabular}

Figure 7 Distribution of non-communicable diseases in primary care visits, 2016-2018.

heart disease in this population. ${ }^{45}$ Besides, we found that the proportions increased and the rankings rose with age for both men and women for ischaemic heart disease, cerebrovascular sequelae and other diseases, whereas the proportion decreased and the ranking dropped with age for acute upper respiratory infection. The prevalence of upper respiratory infection decreased with age in the study conducted by Zhang and Shi from 2008 to 2012. ${ }^{36}$ Huang $e t a l^{25}$ found that tumour was consistently the top inpatient condition for both men and women in specialist visits, and inpatient pregnancy, childbirth and puerperium disorders were the most common conditions for inpatient women. In terms of NCDs and disease systems, Huang $e t a l$ s study indicated that, as age increased, the number and proportion of visits for NCDs also increased. This finding was degree. Wang et $a t^{46}$ found that the prevalence of NCDs increased with age and that women had a higher probability of suffering from NCDs. Visits of patients aged younger than 18 years were previously found to be more likely to be caused by respiratory diseases, oral diseases and skin diseases for both male and female patients, and this finding was echoed by a recent study on specialist visits. ${ }^{36}$ In the present study, the topranked NCD in the 19-39 years age group was diseases of the urogenital system for women and cardiovascular system diseases for men. Compared with women, more men suffered from cardiovascular diseases at an earlier age, which is consistent with previous studies. ${ }^{46}$ One study focusing on the disease composition of inpatients in one CHSC also found that women were more likely than men to suffer from diseases of the urogenital system. ${ }^{47}$ Among the three oldest age groups in the present study (40-59 years, $60-79$ years and $\geq 80$ years), the proportion of visits for cardiovascular and circulatory diseases ranked highest for both men and women. Cardiovascular and circulatory diseases have also shown to rank highly in the disease composition of specialist visits by people in the middle and older age groups. ${ }^{37} 3848$
We compared our findings with those of other countries. Although current research on disease composition does not focus on the field of primary healthcare on a global scale, we still found some similar studies conducted in other countries. Mash $e t a t^{49}$ conducted a morbidity survey of South African primary care, and they found that the top five diagnosed diseases in South Africa were hypertension (12\%), upper respiratory tract infection $(5.3 \%)$, HIV/AIDS (3.9\%), type 2 diabetes $(3.9 \%)$ and TB (3.6\%). Another primary care study conducted in Eastern Cape Province of South Africa showed that the most commonly diagnosed category was respiratory diseases $(23 \%) .^{50}$ Malaria was also highlighted as a key prevention and treatment disease for primary care in many African countries such as Kenya. ${ }^{51}$ Different to the current findings in China, infectious diseases including HIV/AIDS are still an important disease component of primary healthcare visits. NCDs are currently the main diagnosis and treatment diseases in primary healthcare institutions in Shanghai, China, where HIV/AIDS were scarcely detected. Another interesting finding was that the dominant focus on primary care diseases in developed countries including UK, USA, Australia, even Chile and Brazil were psychiatric morbidity, depression, anxiety or mental health, although we did not find the exact proportion of such diseases at the primary care level nationwide. ${ }^{52}{ }^{53}$ Martín-Merino et a $a \tilde{l}^{54}$ proposed that the prevalence of anxiety was $7.2 \%$ based on a cohort study conducted in UK, ${ }^{54}$ Vazquez-Barquero et a $a \bar{l}^{55}$ found the prevalence of mental illness (in attenders) was $33.2 \%$ in northern Spain while Mari et $a p^{\tilde{6}}$ insisted that the hidden psychiatric morbidity ranged from $22 \%$ to $79 \%$ in primary care clinics in the city of Sao Paulo of Brazil. Besides, obesity of the youth was also highlighted in current studies on primary care in advanced countries such as Germany, Canada and Australia, which was less perceived by primary care institutions in China. ${ }^{5758} \mathrm{~A}$ further crosscountry comparison study is worth conducting, focusing 


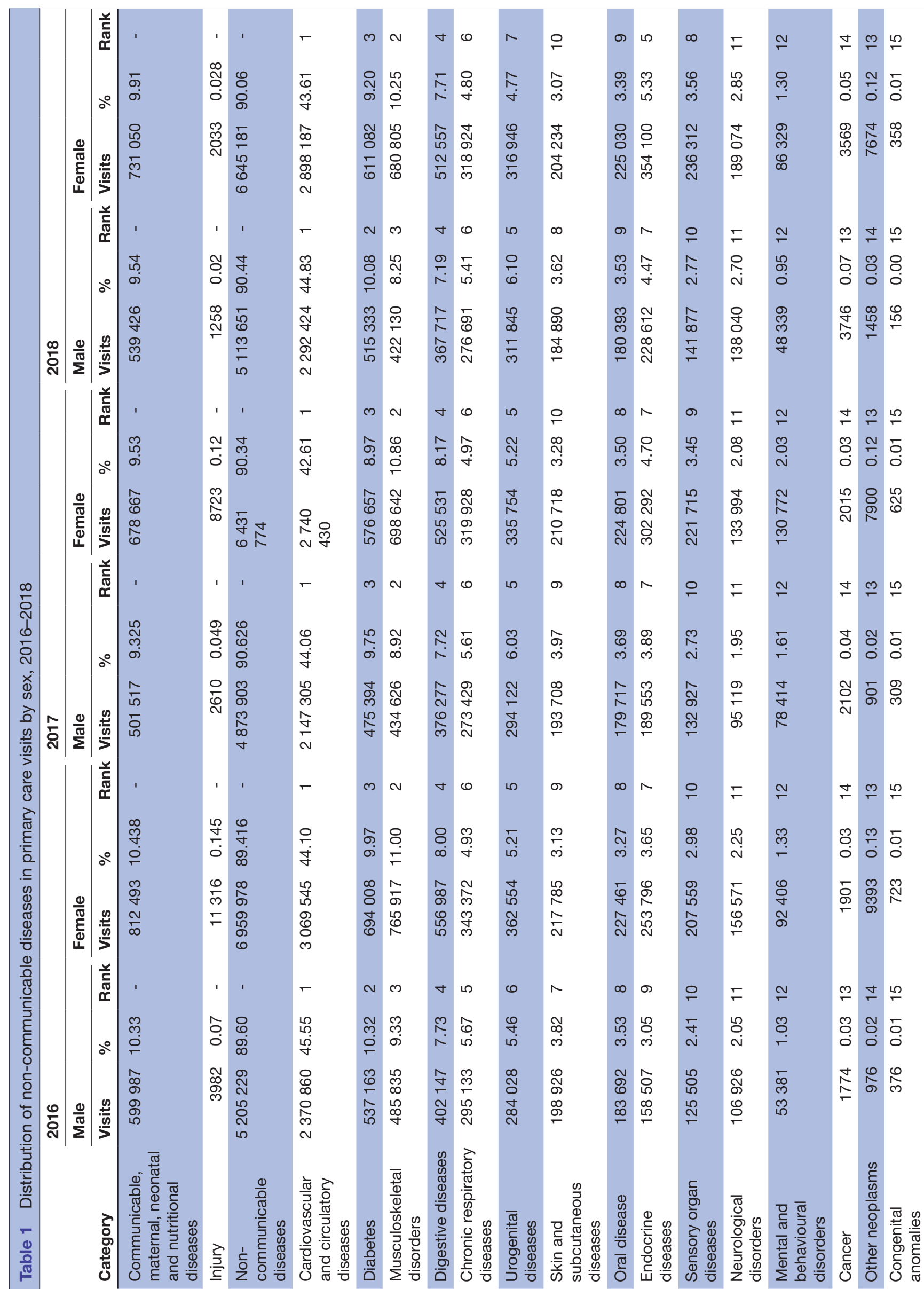




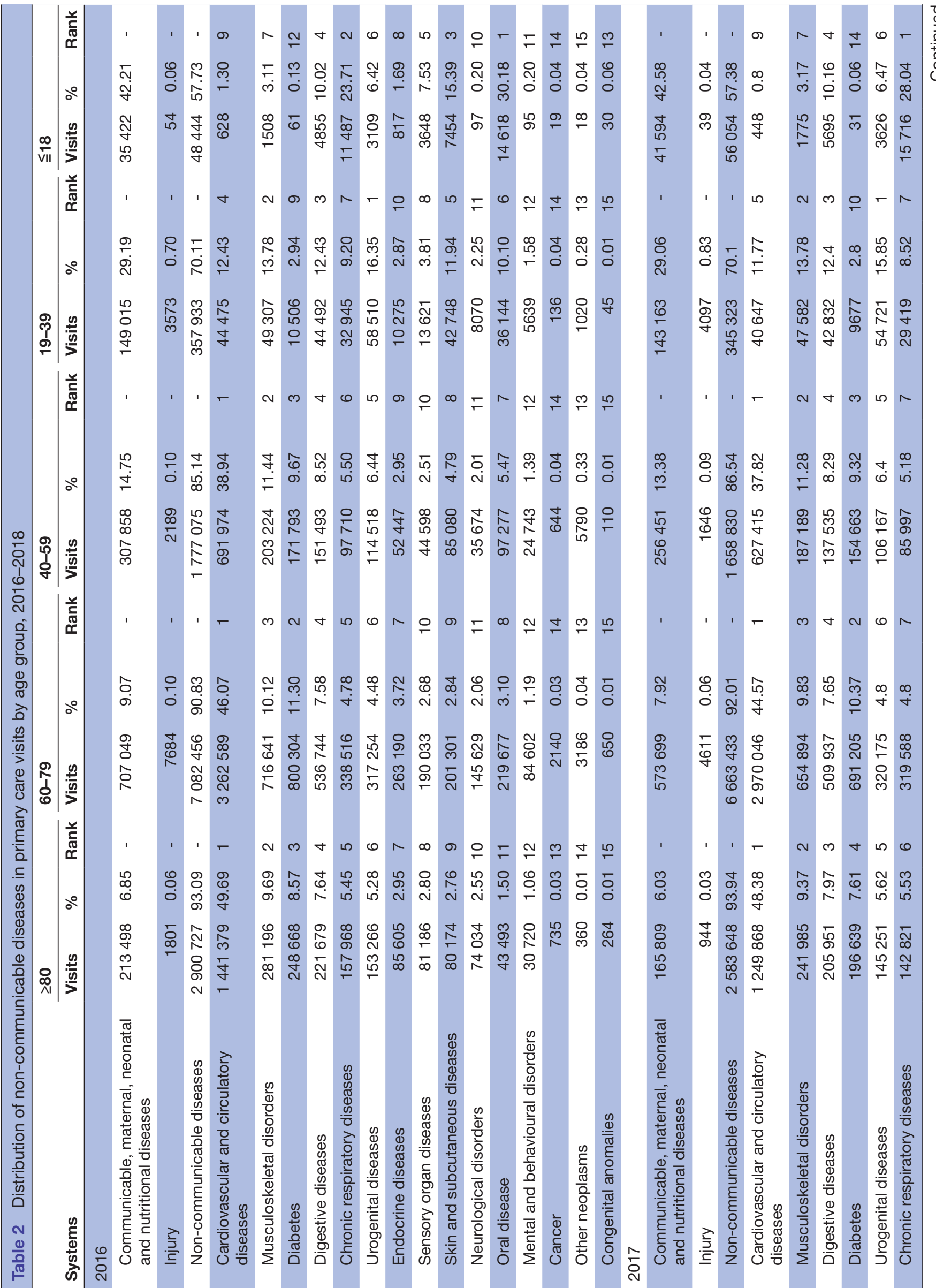




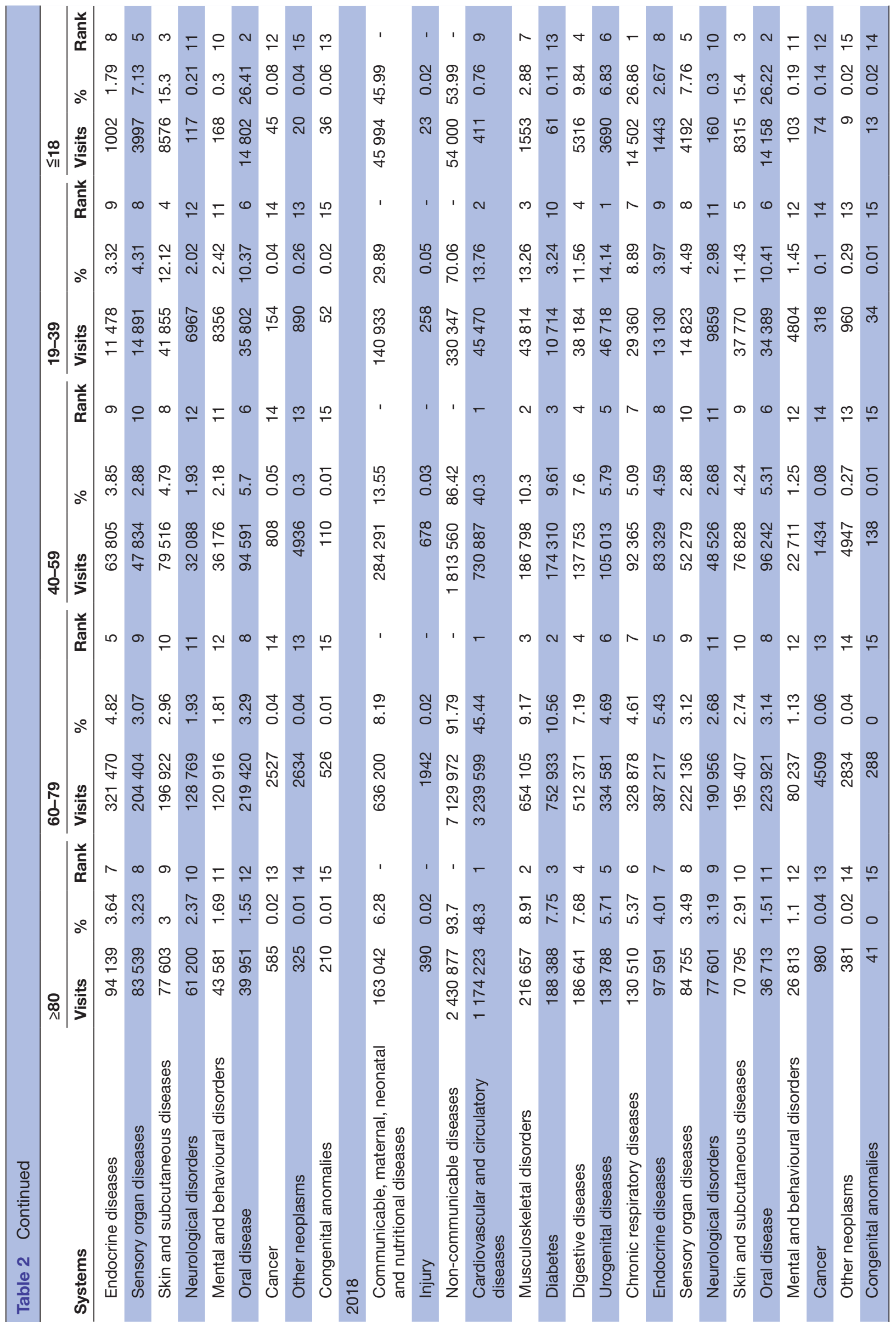


on detailed disease morbidity and its various factors in primary care.

In addition, there are two points worth noting. First, you might have noticed the unusual name of the Z-ICD code. Problems related to medical facilities is one of the Z-ICD diseases, accounting for a large proportion, from around $9 \%$ to $15 \%$. We learnt about the disease diagnosis content included in this code by consulting the information technology section of the Health Commission. Problems related to medical facilities mainly refer to health examination (including occupational exam, child exam, student exam, age $\geq 60$ years exam) and some other problems, and factors influencing health status and contact with health services is a health system category-the proportion of which dropped from around $16 \%$ to $10 \%-$ which is mainly composed of problems related to medical facilities and some other health consultations. These two classifications have a certain impact on our analysis of disease composition. However, as disease diagnosis becomes more precise and standardised, the proportions of these two categories are gradually decreasing. We insist that the decline of these two categories will improve the accuracy of analysis of disease composition, but we also believe that these two categories cannot be zero, as there are health examination cases and undiagnosable cases. Second, NCD-oriented disease intervention is currently the main task of CHSCs in China, at least at the survey site. So, we believe that the current health system matches the morbidity of primary care. However, a problem that cannot be ignored is that mental illnesses are rarely diagnosed in CHSCs. This does not mean that our residents are mentally healthy. On the contrary, stigma prevents residents from taking any measures until their mental health conditions have deteriorated. Psychological problems of residents have been exposed during the COVID-19 outbreak and transmission period, and obviously the FDs of CHSCs still cannot offer psychological consultation, diagnosis and treatment.

\section{CONCLUSION}

There are four main findings of this study. First, primary care mostly covered circulatory system diseases, respiratory system diseases, endocrine/nutritional/metabolic diseases, factors influencing health status and digestive system diseases, indicating the cross-coverage of disease systems with specialised hospitals. Second, the top causes for primary care visits were consistently found to be primary hypertension, chronic ischaemic heart disease, unspecified diabetes mellitus and acute upper respiratory tract infection; this finding differed significantly from previous findings for tertiary hospitals, suggesting an initial functional differentiation between CHSCs and large hospitals. Third, there is an ongoing health transition in China, with NCDs accounting for $90 \%$ of all CHSC visits, underscoring the importance of a well-functioning FD system to deal with future NCD management burden. Fourth, there is significant variation by sex and age, indicating that life-cycle-specific health services-rather than homogenised services-should be provided.

\section{Limitations}

First, all our data were from a single district, although this district is the largest one in Shanghai, with a permanent population of 5.5 million. Second, to include high-quality data from all 47 CHSCs in the district, we were only able to extract data since 2016, so the study lacked comparison with earlier data, and reliable regression models were also impossible to perform. Third, COVID-19 has not yet occurred during our research period, which might change our results to some degree, thus it is necessary to further explore the epidemiological characteristics of primary care visits especially during 2019 and 2020, or even longer. Finally, the data extracted from the EHR were lacking in factors such as education, occupation, income and some regional variables, thus we only analysed the data by age and sex.

\section{Author affiliations}

${ }^{1}$ Heart Failure Department, Shanghai East Hospital affiliated to Tongji University

School of Medicine, Shanghai, China

${ }^{2}$ Pudong Health Information Center, Pudong Institute for Health Development, Shanghai, China

${ }^{3}$ School of Public Health, Shanghai Jiao Tong University School of Medicine, Shanghai, China

${ }^{4}$ School of Medicine, Tongji University, Shanghai, China

${ }^{5}$ Shanghai Tenth People's Hospital, Tongji University School of Medicine, Shanghai, China

Acknowledgements The authors thank the Pudong health information centre for assistance with data collection.

Contributors ZW conceived and designed the study. XW, JS, TS and WY were involved with data collection. $\mathrm{JH}, \mathrm{LZ}$ and $\mathrm{NC}$ analysed the data. $\mathrm{XG}$ and $\mathrm{JH}$ wrote the article.

Funding This work was supported by the National Natural Science Foundation of China $(71744116,71904145,71603182,71804128)$, the National Key Research and Development Program of China (2018YFC2000700, SQ2018YFC130057) and the Shanghai Pujiang Program (2019PJC072).

Competing interests None declared.

Patient consent for publication Not required.

Ethics approval This study was approved by the ethics committees of Shanghai Tenth People's Hospital (ref: 2019-K173-02). The research presented minimal risk of harm to participants, and the study only included primary care visits data collected anonymously.

Provenance and peer review Not commissioned; externally peer reviewed.

Data availability statement The data generated and analysed during the present study are only for academic use and are not publicly available because of patient privacy proctection. However, they are available from the corresponding author ( supercell002@sina.com) for researchers who meet the criteria for access to confidential data.

Supplemental material This content has been supplied by the author(s). It has not been vetted by BMJ Publishing Group Limited (BMJ) and may not have been peer-reviewed. Any opinions or recommendations discussed are solely those of the author(s) and are not endorsed by BMJ. BMJ disclaims all liability and responsibility arising from any reliance placed on the content. Where the content includes any translated material, BMJ does not warrant the accuracy and reliability of the translations (including but not limited to local regulations, clinical guidelines, terminology, drug names and drug dosages), and is not responsible for any error and/or omissions arising from translation and adaptation or otherwise.

Open access This is an open access article distributed in accordance with the Creative Commons Attribution Non Commercial (CC BY-NC 4.0) license, which 
permits others to distribute, remix, adapt, build upon this work non-commercially, and license their derivative works on different terms, provided the original work is properly cited, appropriate credit is given, any changes made indicated, and the use is non-commercial. See: http://creativecommons.org/licenses/by-nc/4.0/.

\section{ORCID iDs}

Jianwei Shi http://orcid.org/0000-0002-3523-6246

Jiaoling Huang http://orcid.org/0000-0003-1975-3937

Zhaoxin Wang http://orcid.org/0000-0002-5022-4497

\section{REFERENCES}

1 Vogel RL, Ackermann RJ. Is primary care physician supply correlated with health outcomes? Int J Health Serv 1998;28:183-96.

2 Shi L. The relationship between primary care and life chances. $J$ Health Care Poor Underserved 1992;3:321-35.

3 Velasco Garrido M, Zentner A, Busse R. The effects of gatekeeping: a systematic review of the literature. Scand J Prim Health Care 2011;29:28-38.

4 Starfield B, Shi L, Macinko J. Contribution of primary care to health systems and health. Milbank Q 2005;83:457-502.

5 Shi L, Macinko J, Starfield B, et al. Primary care, infant mortality, and low birth weight in the states of the USA. J Epidemiol Community Health 2004;58:374-80.

6 Li M, Zhang Y, Lu Y, et al. Factors influencing two-way referral between hospitals and the community in China: a system dynamics simulation model. Simulation 2018;94:765-82.

7 Li L, Zhong C, Mei J, et al. Effect of family practice contract services on the quality of primary care in Guangzhou, China: a cross-sectiona study using PCAT-AE. BMJ Open 2018;8:e021317-8.

8 World Bank. Better health in Africa: experience and lessons learned. Washington, DC: World Bank, 1994

$9 \mathrm{Li} \mathrm{X,} \mathrm{Lu} \mathrm{J,} \mathrm{Hu} \mathrm{S,} \mathrm{et} \mathrm{al.} \mathrm{The} \mathrm{primary} \mathrm{health-care} \mathrm{system} \mathrm{in} \mathrm{China.}$ Lancet 2017;390:2584-94.

10 Yang T, Lei R, Jin X, et al. Supervisor support, Coworker support and Presenteeism among healthcare workers in China: the mediating role of distributive justice. Int J Environ Res Public Health 2019;16:817-9.

11 Huang J, Liu S, He R, et al. Factors associated with residents' contract behavior with family doctors in community health service centers: a longitudinal survey from China. PLoS One 2018;13:e0208200-16.

12 Cheng J-M, Yuan Y-X, Lu W, et al. Primary health care in China: is China's health reform reform for the whole nation? Prim Health Care Res Dev 2017:18:398-403.

13 National Medical Reform Office. Notice on promoting guidance for family doctor contracted services, 2016. Available: http://www.gov. cn/xinwen/2016-06/06/content 5079984.htm

14 Chi-Man W, William C, Chen W, et al. Early appraisal of China's huge and complex health-care reforms. Lacet 2012;379:833-42.

15 Kuang L, Liang Y, Mei J, et al. Family practice and the quality of primary care: a study of Chinese patients in Guangdong Province. Fam Pract 2015:32:557-63.

16 Shi L, Makinen M, Lee D-C, et al. Integrated care delivery and health care seeking by chronically-ill patients - a case-control study of rural Henan Province, China. Int J Equity Health 2015;14:1-15.

17 Chao J, Xie W, Yang Y, et al. The effect of integrated health management model on the satisfaction among Chinese elderly. Arch Gerontol Geriatr 2013;57:27-31.

$18 \mathrm{Li} \mathrm{L}$. Evaluation of healthcare reform in Xiamen. Modern Health Management 2016;14:16.

$19 \mathrm{Li} \mathrm{H}, \mathrm{Wu} \mathrm{Z}$, Hui X, et al. Impact of local health insurance schemes on primary care management and control of hypertension: a crosssectional study in Shenzhen, China. BMJ Open 2019;9:e031098.

20 Huang J, Lu W, Wang L, et al. A preliminary effect analysis of family doctor and medical insurance payment coordination reform in Changning district of Shanghai, China. BMC Fam Pract 2019;20:1-10.

21 Liu Y, Yang G, Zeng Y, et al. Policy dialogue on China's changing burden of disease. The Lancet 2013;381:1961-2.

22 Sohrabi C, Alsafi Z, O'Neill N, O'Neill N, et al. World Health organization declares global emergency: a review of the 2019 nove coronavirus (COVID-19). Int J Surg 2020;76:71-6.

23 WHO. Global status report on noncommunicable diseases. Geneva, Switzerland: WHO Press, 2010.

24 Zhou M, Wang $\mathrm{H}$, Zeng $\mathrm{X}$, et al. Mortality, morbidity, and risk factors in China and its provinces, 1990-2017: a systematic analysis for the global burden of disease study 2017. Lancet 2019;394:1145-58.

25 Huang DD, Zou MR, Zhao YC, et al. Analysis of spectrum of disease composition of inpatients in a grade a tertiary hospital for 10 years. Jiangsu Health System Management 2019;30:1502-10.
26 Xia QH, Jiang Y, Jia H, et al. Analysis on epidemic characteristics and health burden of 6205 hospitalized injury cases in Changning district, Shanghai. Chinese Journal of Health Education 2011;27:412-37.

27 Huang Y-M, Xu D, Long J, et al. Spectrum of chronic kidney disease in China: a national study based on hospitalized patients from 2010 to 2015. Nephrology 2019;24:725-36.

28 Chen S-X, Fan K, Leung L-P. Epidemiological characteristics and disease spectrum of emergency patients in two cities in China: Hong Kong and Shenzhen. World J Emerg Med 2020;11:48-53.

29 Ji D, Chen G, Fu Y, et al. The 10-year chronic liver disease spectrum evolution in China: experience from the largest tartiary special hospital with 21382 liver biopsy cases. J Hepatol 2018;68:S155-6.

30 Huang XL, Chen ZX, Yang YB, et al. Current status and new ideas in research on disease spectrum of inpatients in China. Chinese Journal of Health Statistics 2012;29:466.

31 Shanghai Statistic Commission. Shanghai statistical Yearbook 20122017. China Statistics Press, 2018.

32 Shanghai Pudong New Area Statistic Commission. Shanghai Pudong new area statistic Yearbook 2017. China Statistics Press, 2017.

33 National Center for Cardiovascular Diseases of China. Report on cardiovascular disease in China. Beijing Encyclopedia of China Publishing House; 2018.

34 Chen N, Shi J, Huang J, et al. Impact of air pollutants on pediatric admissions for Mycoplasma pneumonia: a cross-sectional study in Shanghai, China. BMC Public Health 2020;20:1-9.

35 Guo WW. Analysis of changes in the disease spectrum of inpatients in a hospital from 2010 to 2013. Chinese J Hospital Statistics 2014;21:471-2.

36 Zhang J, Shi JJ. Analysis of changes in the disease spectrum of inpatients in a hospital from 2008 to 2012. Chinese $J$ Hospital Statistics 2013;20:397-8.

37 Sha LN. Analysis of the top 10 diseases of inpatients from 2005 to 2009. Medical Information 2010;23:2783-5

38 Zhang YH, Wang ZP. Analysis of inpatient disease spectrum changes, 2007-2011. Chinese J Hospital Statistics 2012;19:274-6.

39 Zhang X, Zhou X, Huang X, et al. The analysis of the disease spectrum in China. Biomed Res Int 2014;2014:1-8.

40 Yang G, Kong L, Zhao W, et al. Emergence of chronic noncommunicable diseases in China. Lancet 2008;372:1697-705.

$41 \mathrm{Yu} \mathrm{D}$, Shi J, Zhang $\mathrm{H}$, et al. Identifying patterns of non-communicable diseases in developed eastern coastal China: a longitudinal study of electronic health records from 12 public hospitals. BMJ Open 2017;7:e016007.

42 WHO. Primary health care: now more than ever. Geneva, Switzerland WHO Press; 2008.

43 Kane J, Landes M, Carroll C, et al. A systematic review of primary care models for non-communicable disease interventions in subSaharan Africa. BMC Fam Pract 2017;18:1-12

44 Abbey SE, Stewart DE. Gender and psychosomatic aspects of ischemic heart disease. J Psychosom Res 2000;48:417-23.

45 Ciambrone G, Kaski JC. Gender differences in the treatment of chronic ischemic heart disease: prognostic implications. Fundam Clin Pharmacol 2010;24:707-10.

46 Wang YQ, HH L, YP L, et al. A status survey on inpatient diseases constitution and hospitalization expenses in Luxi Township Health Center, Yongxin County of Jiangxi Province in 2010. Chinese $J$ Evidence-Based Med 2012;12:16-24.

47 Teng SM. Analysis of the composition of the top 10 diseases of inpatients in a primary hospital, 2008 - 2012. Chinese $J$ Hospital Statistics 2013;20:315-7.

48 Guo W, Mei W, Fang G, et al. Analysis on disease spectrum of inpatients in a City from 2014 to 2016. Chinese Medical Record 2018;19:62-5.

49 Mash B, Fairall L, Adejayan O, et al. A morbidity survey of South African primary care. PLoS One 2012;7:e32358.

50 Brueton V, Yogeswaran P, Chandia J, et al. Primary care morbidity in eastern cape province. S Afr Med J 2010;100:309-12.

51 O'Meara WP, Noor A, Gatakaa H, et al. The impact of primary health care on malaria morbidity-defining access by disease burden. Trop Med Int Health 2009;14:29-35.

52 Gunn JM, Ayton DR, Densley K, et al. The association between chronic illness, multimorbidity and depressive symptoms in an Australian primary care cohort. Soc Psychiatry Psychiatr Epidemiol 2012;47:175-84

53 Skapinakis P, Araya R. Common somatic symptoms, causal attributions of somatic symptoms and psychiatric morbidity in a cross-sectional community study in Santiago, Chile. BMC Res Notes 2011;4:1-5

54 Martín-Merino E, Ruigómez A, Wallander M-A, et al. Prevalence, incidence, morbidity and treatment patterns in a cohort of patients diagnosed with anxiety in UK primary care. Fam Pract 2010;27:9-16. 
55 Vázquez-Barquero JL, García J, Simón JA, et al. Mental health in primary care. An epidemiological study of morbidity and use of health resources. Br J Psychiatry 1997;170:529-35.

56 Mari JdeJ, lacoponi E, Williams $\mathrm{P}$, et al. Detection of psychiatric morbidity in the primary medical care setting in Brazil. Rev Saude Publica 1987;21:501-7.
57 Balkau B, Deanfield JE, Després JP, et al. Idea: a study of waist circumference, cardiovascular disease and diabetes in 168,000 primary care patients in 63 countries. Circulation 2007;116:1942-51.

58 Bramlage $\mathrm{P}$, Wittchen $\mathrm{H}-\mathrm{U}$, Pittrow D, et al. Recognition and management of overweight and obesity in primary care in Germany. Int J Obes Relat Metab Disord 2004;28:1299-308. 